\title{
THE FIRST INTEGRALS AND THEIR LIE ALGEBRA OF THE MOST GENERAL AUTONOMOUS HAMILTONIAN OF THE FORM $H=T+V$ POSSESSING A LAPLACE-RUNGE-LENZ VECTOR
}

\author{
V. M. GORRINGE and P. G. L. LEACH ${ }^{1}$
}

(Received 9 April 1990; revised 6 May 1992)

\begin{abstract}
In two dimensions it is found that the most general autonomous Hamiltonian possessing a Laplace-Runge-Lenz vector is $H=\frac{1}{2} p \cdot p-\mu r^{-1}-\alpha r^{-1 / 2} \cos ((\theta-\beta) / 2)$. The Poisson bracket of the two components of this vector leads to a third firstintegral, cubic in the momenta. The Lie algebra of the three integrals under the operation of the Poisson bracket closes, and is shown to be $s o(3)$ for negative energy and $s o(2,1)$ for positive energy. In the case of zero energy, the algebra is $W(3,1)$. The result does not have a three-dimensional analogue, apart from the usual Kepler problem.
\end{abstract}

\section{Introduction}

Existence of conserved vectors of Laplace-Runge-Lenz-type (the most appropiate name for this type of vector is arguable [3], [4]) for all central-force planar motions was demonstrated some years ago by Fradkin [2], and for all planar motions by Yoshida [17] more recently. Explicit expressions have been found for many types of two- and three-dimensional equations of motion [9], [10], [14], but there are only two autonomous Hamiltonians of the type $H=T+V$ for which they have been found, the usual Kepler problem Hamiltonian and

$$
H=\frac{1}{2} \mathbf{p} \cdot \mathbf{p}-\mu / r-\alpha r^{-1 / 2} \cos ((\theta-\beta) / 2),
$$

where $\mu, \alpha$ and $\beta$ are constants, $(r, \theta)$ are plane polar co-ordinates and $p$ is the canonically conjugate momentum (the mass is set at unity). To

${ }^{1}$ Centre for Nonlinear Studies and Department of Computational and Applied Mathematics, University of the Witwatersrand, P.O. WITS, 2050 South Africa.

(C) Copyright Australian Mathematical Society 1993, Serial-fee code 0334-2700/93 
the best of our knowledge the Laplace-Runge-Lenz vector for (1.1) was first derived by Sen [13], who used Bertrand's method [15] with the co-ordinates and momenta written in terms of the complex conjugate variables $z, \bar{z}$ and $p_{z}, p_{z}$. The Hamiltonian (1.1) and the conserved vector

$$
\mathbf{J}=\mathbf{p} \times \mathbf{L}-\mu \hat{\mathbf{r}}-\alpha r^{1 / 2} \sin ((\theta-\beta) / 2) \hat{\theta},
$$

where $\mathbf{L}$ is the angular momentum $\mathbf{r} \times \mathbf{p}$ and here, as elsewhere in this paper, an overcaret denotes a unit vector, are found after some manipulation when the requirement of reality is imposed upon his results [13], equations $(3.12,3.14)$. Sen [13] also showed the existence of a first integral which is cubic in the momenta, and stated that the Lie algebra of the three invariants under the operation of taking the Poisson bracket was isomorphic to $s o(3)$ (negative energy) or $s o(2,1)$ (positive energy), just as for the Kepler problem in two dimensions. The Hamiltonian (1.1) and conserved vector (1.2) were also found as a particular result of a more general problem by Gorringe and Leach [5]. They adapted the method of direct vectorial manipulation of the equation of motion which Collinson [1] had employed to find the LaplaceRunge-Lenz vector for the Kepler problem. They found that the equation of motion

$$
\ddot{\mathbf{r}}+\left[\frac{U^{\prime \prime}(\theta)+U(\theta)}{r^{2}}+\frac{2 V^{\prime}(\theta)}{r^{3 / 2}}\right] \hat{\mathbf{r}}+\frac{V(\theta)}{r^{3 / 2}} \hat{\theta}=0
$$

possesses the conserved vector

$$
\mathbf{J}=\dot{\mathbf{r}} \times \mathbf{L}-U(\theta) \hat{\mathbf{r}}-\left[U^{\prime}(\theta)+2 r^{1 / 2} V(\theta)\right] \hat{\theta},
$$

where $U$ and $V$ are arbitrary functions of $\theta$. The results (1.1) and (1.2) follow from requiring that the force in (1.3) be derivable from a potential.

Although we do not wish to detract from the technical excellence of Sen's work, it is very technical, and in this note we offer an alternative derivation of (1.1), (1.2) and the cubic invariant, provide an elegant treatment of the Lie algebra of the invariants and attempt to correct a misconception in the Appendix of Sen's paper. (The misconception was also held by one of the present authors [7] in his younger days.) The peculiarity of the results (1.1) and (1.2) to two dimensions is shown in an Appendix. The method of the derivation of the integrals is as follows. We take a two dimensional Hamiltonian of the form

$$
H=\frac{1}{2} \mathbf{p} \cdot \mathbf{p}+V(r, \theta)
$$

and assume that it possesses a Laplace-Runge-Lenz vector of the form

$$
\mathbf{J}=\mathbf{p} \times \mathbf{L}+\mathbf{g}(r, \boldsymbol{\theta}),
$$


where the potential $V(r, \theta)$ and vector $\mathbf{g}(r, \theta)$ are to be determined. (For the Kepler problem $V=-\mu r^{-1}$ and $\mathbf{g}=\mu \hat{\mathbf{r}}$.) To determine $V$ and $\mathbf{g}$ we take the Poisson bracket of $\mathbf{J}$ with $H$ and demand that it be zero. We then use Poisson's theorem [16] to find the third integral. The algebra of the integrals is then established.

\section{Determination of the potential and vector}

In plane polar co-ordinates, the Hamiltonian (1.5) is

$$
H=\frac{1}{2}\left(p_{r}^{2}+\frac{p_{\theta}^{2}}{r^{2}}\right)+V(r, \theta)
$$

and the Cartesian components of the vector (1.6) are

$$
\begin{aligned}
& J_{1}=p_{r} p_{\theta} \sin \theta+\frac{p_{\theta}^{2}}{r} \cos \theta+f(r, \theta), \\
& J_{2}=-p_{r} p_{\theta} \cos \theta+\frac{p_{\theta}^{2}}{r} \sin \theta+g(r, \theta) .
\end{aligned}
$$

The requirements that $\left[J_{1}, H\right]_{P B}$ and $\left[J_{2}, H\right]_{P B}$ be zero lead to the following relations between $f, g$ and $V$ :

$$
\begin{aligned}
& \frac{\partial f}{\partial r}=\sin \theta \frac{\partial V}{\partial \theta}, \\
& \frac{\partial f}{\partial \theta}=r^{2} \sin \theta \frac{\partial V}{\partial r}+2 r \cos \frac{\partial V}{\partial \theta}, \\
& \frac{\partial g}{\partial r}=-\cos \theta \frac{\partial V}{\partial \theta}, \\
& \frac{\partial g}{\partial \theta}=-r^{2} \cos \theta \frac{\partial V}{\partial r}+2 r \sin \theta \frac{\partial V}{\partial \theta} .
\end{aligned}
$$

Consistency between the pairs of equations $(2.4,5)$ and $(2.6,7)$ leads to two equations for $V$ which may be combined in two ways to give

$$
\begin{aligned}
& \frac{\partial^{2} V}{\partial \theta^{2}}=\frac{\partial}{\partial r}\left(r^{2} \frac{\partial V}{\partial r}\right), \\
& \frac{\partial V}{\partial \theta}=2 \frac{\partial}{\partial r}\left(r \frac{\partial V}{\partial \theta}\right) .
\end{aligned}
$$

The solution of these two equations is

$$
V(r, \theta)=-\frac{\mu}{r}-\frac{\alpha \cos ((\theta-\beta) / 2)}{r^{1 / 2}},
$$


where $\mu, \alpha$ and $\beta$ are constants of integration. (An additive constant has been ignored.) This is the potential in (1.1). Returning to equations (2.4-7) we substitute $V$ from (2.10), and find that

$$
\begin{gathered}
f(r, \theta)=-\mu \cos \theta+\alpha r^{1 / 2} \sin ((\theta-\beta) / 2) \sin \theta, \\
g(r, \theta)=-\mu \sin \theta-\alpha r^{1 / 2} \sin ((\theta-\beta) / 2) \cos \theta
\end{gathered}
$$

so that (2.2) and (2.3) are

$$
\begin{aligned}
& J_{1}=p_{r} p_{\theta} \sin \theta+\frac{p_{\theta}^{2}}{r} \cos \theta-\mu \cos \theta+\alpha r^{1 / 2} \sin ((\theta-\beta) / 2) \sin \theta, \\
& J_{2}=-p_{r} p_{\theta} \cos \theta+\frac{p_{\theta}^{2}}{r} \sin \theta-\mu \sin \theta-\alpha r^{1 / 2} \sin ((\theta-\beta) / 2) \cos \theta
\end{aligned}
$$

and the Laplace-Runge-Lenz vector (1.2) is recovered.

\section{The third integral and the Lie algebra of the invariants}

Poisson's theorem [16] states that, provided Jacobi's identity is satisfied, the Poisson bracket of two first integrals is itself a first integral. If we take the Poisson bracket of $J_{1}$ and $J_{2}$, we find, after not a little algebra, that

$$
\begin{aligned}
{\left[J_{1}, J_{2}\right]_{P B} } & =\frac{\partial J_{1}}{\partial r} \frac{\partial J_{2}}{\partial p_{r}}+\frac{\partial J_{1}}{\partial \theta} \frac{\partial J_{2}}{\partial p_{\theta}}-\frac{\partial J_{1}}{\partial p_{r}} \frac{\partial J_{2}}{\partial r}-\frac{\partial J_{1}}{\partial p_{\theta}} \frac{\partial J_{2}}{\partial \theta} \\
& =-2 p_{\theta} H-p_{r} \alpha r^{1 / 2} \sin ((\theta-\beta) / 2)-p_{\theta} \alpha r^{-1 / 2} \cos ((\theta-\beta) / 2)
\end{aligned}
$$

and we define the third integral to be

$$
I=2 p_{\theta} H+p_{r} \alpha r^{1 / 2} \sin ((\theta-\beta) / 2)+p_{\theta} \alpha r^{-1 / 2} \cos ((\theta-\beta) / 2) .
$$

We then compute the Poisson brackets of $J_{1}$ and $J_{2}$ with $I$ and have

$$
\begin{aligned}
& {\left[J_{1}, J_{2}\right]_{P B}=-I,} \\
& {\left[J_{1}, I\right]_{P B}=-2 H J_{2}-\frac{1}{2} \alpha^{2} \sin \beta,} \\
& {\left[J_{2}, I\right]_{P B}=2 H J_{1}+\frac{1}{2} \alpha^{2} \cos \beta .}
\end{aligned}
$$

The bracket relations (3.2-4) are not very tidy. For negative energy we define

$$
\begin{aligned}
& A_{-}=\frac{1}{\sqrt{-2 H}}\left(J_{1}+\frac{\alpha^{2} \cos \beta}{4 H}\right), \\
& B_{-}=\frac{1}{\sqrt{-2 H}}\left(J_{2}+\frac{\alpha^{2} \sin \beta}{4 H}\right), \\
& C_{-}=\frac{I}{2 H} .
\end{aligned}
$$


Using (3.2-4) we see that

$$
\begin{aligned}
& {\left[A_{-}, B_{-}\right]_{P B}=C_{-},} \\
& {\left[B_{-}, C_{-}\right]_{P B}=A_{-},} \\
& {\left[C_{-}, A_{-}\right]_{P B}=B_{-},}
\end{aligned}
$$

which is immediately recognisable as the Lie algebra $s o(3)$. For positive energy we define

$$
\begin{aligned}
& A_{+}=\frac{1}{\sqrt{2 H}}\left(J_{1}+\frac{\alpha^{2} \cos \beta}{4 H}\right), \\
& B_{+}=\frac{1}{\sqrt{2 H}}\left(J_{2}+\frac{\alpha^{2} \sin \beta}{4 H}\right), \\
& C_{+}=\frac{I}{2 H},
\end{aligned}
$$

so that

$$
\begin{aligned}
& {\left[A_{+}, B_{+}\right]_{P B}=-C_{+},} \\
& {\left[B_{+}, C_{+}\right]_{P B}=A_{+},} \\
& {\left[C_{+}, A_{+}\right]_{P B}=B_{+},}
\end{aligned}
$$

which are the Poisson bracket relations for the non-compact Lie algebra so $(2,1)$.

It is well known for the Kepler problem that the angular momentum, Laplace-Runge-Lenz vector, and the Hamiltonian are not independent, but are related according to the equation

$$
J^{2}=2 L^{2} H+\mu^{2} \text {. }
$$

A similar relationship is found for this problem. After some manipulation, we find that

$$
\frac{1}{2 H}\left\{\left(J_{1}+\frac{\alpha^{2} \cos \beta}{4 H}\right)^{2}+\left(J_{2}+\frac{\alpha^{2} \sin \beta}{4 H}\right)^{2}-\left(\mu-\frac{\alpha^{2}}{4 H}\right)^{2}\right\}=\left(\frac{I}{2 H}\right)^{2} \text {. }
$$

The relationship (3.10) is not very neat. If we define

$$
\begin{gathered}
\mu_{-}=\frac{1}{\sqrt{-2 H}}\left(\mu-\frac{\alpha^{2}}{4 H}\right), \\
\mu_{+}=\frac{1}{\sqrt{2 H}}\left(\mu-\frac{\alpha^{2}}{4 H}\right)
\end{gathered}
$$

for negative and positive energies respectively, (3.10) can be rewritten as

$$
\mu_{-}^{2}=A_{-}^{2}+B_{-}^{2}+C_{-}^{2}
$$


for negative energies and

$$
\mu_{+}^{2}=A_{+}^{2}+B_{+}^{2}-C_{+}^{2}
$$

for positive energies. Equation (3.12) represents a sphere which is naturally associated with $s o(3)$ symmetry, and (3.13) a hyperboloid of one sheet, the natural geometric object associated with $s o(2,1)$ symmetry.

When $H$ takes on the particular value of zero, the analysis following (3.4) is no longer valid. The third integral is now

$$
I=p_{r} \alpha r^{1 / 2} \sin ((\theta-\beta) / 2)+p_{\theta} \alpha r^{-1 / 2} \cos ((\theta-\beta) / 2)
$$

and is not a true first integral but, rather, a configurational invariant (cf. Hall [6] and Sarlet et al. [12]), since it is invariant only for the particular value of $H$. If we introduce the unit vector $\hat{\beta}$ defined by

$$
\hat{\beta}=\hat{\jmath}_{1} \cos \beta+\hat{\jmath}_{2} \sin \beta,
$$

where $\hat{\jmath}_{1}$ and $\hat{\jmath}_{2}$ are the unit vectors along which $J_{1}$ and $J_{2}$ lie respectively, we find, after a little manipulation, that

$$
\mathbf{J} \cdot \hat{\beta}=I^{2} \alpha^{-2}-\mu
$$

i.e.

$$
J_{1} \cos \beta+J_{2} \sin \beta=I^{2} \alpha^{-2}-\mu .
$$

Equation (3.16) describes the natural geometric object associated with the case $H=0$. It is a right parabolic cylinder with axis of symmetry given by $\hat{\boldsymbol{\beta}}$.

We note that here there is a departure from the standard Kepler problem. In the Kepler problem, when $H=0,(3.9)$ gives $J= \pm \mu$, which defines a plane. This is no longer the case, as can be seen from (3.16). This is reflected in the algebra. If we define

$$
\begin{aligned}
& A_{0}=-J_{1} \sin \beta+J_{2} \cos \beta, \\
& B_{0}=2 I \alpha^{-2}, \\
& C_{0}=1
\end{aligned}
$$

( $J_{1} \cos \beta+J_{2} \sin \beta$ cannot be used as this is a function of $I$ through (3.16)), the Poisson bracket relations are

$$
\begin{aligned}
& {\left[A_{0}, B_{0}\right]_{P B}=C_{0},} \\
& {\left[B_{0}, C_{0}\right]_{P B}=0,} \\
& {\left[C_{0}, A_{0}\right]_{P B}=0}
\end{aligned}
$$


which are those of the Weyl algebra $W(3,1)$. The standard Kepler problem has the algebra $E(2)$ when $H=0$.

When $H \neq 0$, the Hamiltonian can be expressed as a function of the three first integrals, $J_{1}, J_{2}$ and $I$, thereby making the symmetry of the system explicit. A few elementary manipulations of (3.10) yield

$$
H=\frac{1}{2}\left(J^{2}-\mu^{2}\right)^{-1}\left[I^{2}-\alpha^{2}\left(J_{1} \cos \beta+J_{2} \sin \beta+\mu\right)\right] .
$$

As is well known, the first integrals of a Hamiltonian define infinitesimal transformations of the canonical variables. If $K$ is a first integral and $\varepsilon$ the infinitesimal parameter,

$$
\delta q_{i}=\varepsilon \frac{\partial K}{\partial p_{i}}, \quad \delta p_{i}=-\varepsilon \frac{\partial K}{\partial q_{i}} .
$$

However, these expressions need not convey much, if any, meaning. For example, if we take $C_{+}$and $r$,

$$
\begin{aligned}
\delta r=\frac{\varepsilon}{2 H^{2}}\{ & \left(-J_{1} \sin \theta+J_{2} \cos \theta\right) \frac{\alpha \cos ((\theta-\beta) / 2)}{r^{1 / 2}} \\
& \left.+H \alpha r^{1 / 2} \sin ((\theta-\beta) / 2)-p_{r}^{2}+\frac{\alpha \cos ((\theta-\beta) / 2)}{r^{1 / 2}}\right\}
\end{aligned}
$$

which appears to be rather meaningless. The expressions for the other variables and integrals are just as bad. We should point out that in the case of the standard Kepler problem, the situation is not much better. It is true that the infinitesimal transformations generated by $p_{\theta}$ are simple, but the others are not. For example the integral corresponding to $A_{+}$gives for $r$

$$
\delta r=\frac{\varepsilon}{(2 H)^{3 / 2}}\left\{J_{2} \frac{p_{\theta}}{r}+\mu p_{r} \cos \theta\right\},
$$

where $J_{2}$ is now the component of the Laplace-Runge-Lenz vector of the Kepler problem.

\section{Lie point symmetries of the second-order differential equation}

The second-order differential equation derivable from the Hamiltonian $(1.1)$ is

$$
\ddot{\mathbf{r}}+\left(\frac{\mu}{r^{2}}+\frac{\frac{1}{2} \alpha \cos ((\theta-\beta) / 2)}{r^{3 / 2}}\right) \hat{\mathbf{r}}+\frac{\frac{1}{2} \alpha \sin ((\theta-\beta) / 2)}{r^{3 / 2}} \hat{\theta}=0 .
$$

Sen [13] in the Appendix to that paper calculated the Lie point symmetries associated with (4.1), and found only the single symmetry

$$
G=\frac{\partial}{\partial t}
$$


the generator of time translations. He states that (4.2) only leads to the conservation of energy. However, Leach [8], in his study of the Kepler problem through its Lie point symmetries, (see also [11]) showed, amongst other results, that the energy (i.e., Hamiltonian), angular momentum and the components of the Laplace-Runge-Lenz vector can all be obtained from the generator of time translations. This should not come as a surprise, as they are all autonomous invariants.

If $G$ is a symmetry of a differential equation

$$
E(t, \mathbf{r}, \dot{\mathbf{r}}, \ddot{\mathbf{r}})=0
$$

and $I$ is a first integral of (4.3) associated with $G$, then $I$ satisfies the two equations

$$
\begin{gathered}
G^{[1]} I=0, \\
\left.\frac{d I}{d t}\right|_{E=0}=0,
\end{gathered}
$$

where $G^{[1]}$ is the first extension of $G$. If, in plane polar co-ordinates, $G$ is given by

$$
G=\tau(t, r, \theta) \frac{\partial}{\partial t}+\xi(t, r, \theta) \frac{\partial}{\partial r}+\eta(t, r, \theta) \frac{\partial}{\partial \theta},
$$

then

$$
G^{[1]}=G+(\dot{\xi}-\dot{r} \dot{\tau}) \frac{\partial}{\partial \dot{r}}+(\dot{\eta}-\dot{\theta} \dot{\tau}) \frac{\partial}{\partial \dot{\theta}} .
$$

With $G$ given by (4.2), (4.4) becomes

$$
\frac{\partial I}{\partial t}+0 \frac{\partial I}{\partial r}+0 \frac{\partial I}{\partial \theta}+0 \frac{\partial I}{\partial \dot{r}}+0 \frac{\partial I}{\partial \dot{\theta}}=0
$$

the characteristics of which are obtained from the solution of the associated Lagrange's system

$$
\frac{d t}{1}=\frac{d r}{0}=\frac{d \theta}{0}=\frac{d \dot{r}}{0}=\frac{d \dot{\theta}}{0}
$$

and are

$$
\begin{array}{ll}
u_{1}=r, & v_{1}=\dot{r}, \\
u_{2}=\theta, & v_{2}=\dot{\theta} .
\end{array}
$$

In terms of these characteristics, (4.5) becomes

Since

$$
\dot{u}_{1} \frac{\partial I}{\partial u_{1}}+\dot{u}_{2} \frac{\partial I}{\partial u_{2}}+\dot{v}_{1} \frac{\partial I}{\partial v_{1}}+\dot{v}_{2} \frac{\partial I}{\partial v_{2}}=0
$$

$$
\begin{array}{ll}
\dot{u}_{1}=v_{1}, & \dot{v}_{1}=u_{1} v_{2}^{2}-\frac{\mu}{u_{1}^{2}}-\frac{\frac{1}{2} \alpha \cos \left(\left(u_{2}-\beta\right) / 2\right)}{u_{1}^{3 / 2}}, \\
\dot{u}_{2}=v_{2}, & \dot{v}_{2}=-\frac{2 v_{1} v_{2}}{u_{1}}-\frac{\frac{1}{2} \alpha \sin \left(\left(u_{2}-\beta\right) / 2\right)}{u_{1}^{5 / 2}},
\end{array}
$$


the characteristics of (4.11) are found from

$$
\begin{aligned}
\frac{d u_{1}}{v_{1}} & =\frac{d u_{2}}{v_{2}}=\frac{d v_{1}}{u_{1} v_{2}^{2}-\mu / u_{1}^{2}-\frac{1}{2} \alpha \cos \left(\left(u_{2}-\beta\right) / 2\right) u_{1}^{-3 / 2}} \\
& =\frac{d v_{2}}{-2 v_{1} v_{2} / u_{1}-\frac{1}{2} \alpha \sin \left(\left(u_{2}-\beta\right) / 2\right) u_{1}^{-5 / 2}} .
\end{aligned}
$$

If we number the $i$ th element of (4.13) as (4.13.i), $i=1,4$, then the combination

$$
\begin{aligned}
& \left(u_{1} v_{2}^{2}+\mu / u_{1}^{2}+\frac{1}{2} \alpha \cos \left(\left(u_{2}-\beta\right) / 2\right) u_{1}^{-3 / 2}\right) \cdot(4 \cdot 13 \cdot 1) \\
& \quad+\frac{1}{2} \alpha \sin \left(\left(u_{2}-\beta\right) / 2\right) u_{1}^{-1 / 2} \cdot(4 \cdot 13.2)+v_{1} \cdot(4 \cdot 13.3)+u_{1}^{2} v_{2}
\end{aligned}
$$

gives

$$
\frac{d\left[\left(v_{1}^{2}+u_{1}^{2} v_{2}^{2}\right) / 2-\mu / u_{1}-\alpha \cos \left(\left(u_{2}-\beta\right) / 2\right) u_{1}^{-1 / 2}\right]}{0}
$$

so that the term in crochets is a characteristic and so a first integral, the energy. In a similar fashion the components of the Laplace-Runge-Lenz vector are obtained by the combination

$$
\begin{aligned}
& \left(2 u_{1} v_{1} v_{2} \sin u_{2}+3 u_{1}^{2} v_{2}^{2} \cos u_{2}+\frac{1}{2} \alpha u_{1}^{-1 / 2} \sin \left(\left(u_{2}-\beta\right) / 2\right) \sin u_{2}\right) \cdot(4.13 .1) \\
& +\left(u_{1}^{2} v_{1} v_{2} \cos u_{2}-u_{1}^{3} v_{2}^{2} \sin u_{2}+\mu \sin u_{2}+\frac{1}{2} \alpha u_{1}^{1 / 2} \cos \left(\left(u_{2}-\beta\right) / 2\right) \sin u_{2}\right. \\
& \left.+\alpha u_{1}^{1 / 2} \sin \left(\left(u_{2}-\beta\right) / 2\right) \cos u_{2}\right) \cdot(4.13 .2)+\left(u_{1}^{2} v_{2} \sin u_{2}\right) \cdot(4.13 .3) \\
& +\left(u_{1}^{2} v_{1} \sin u_{2}+2 u_{1}^{3} v_{2} \cos u_{2}\right) \cdot(4.13 .4)
\end{aligned}
$$

for $J_{1}$ and

$$
\begin{aligned}
& \left(-2 u_{1} v_{1} v_{2} \cos u_{2}+3 u_{1}^{2} v_{2}^{2} \sin u_{2}-\frac{1}{2} \alpha u_{1}^{-1 / 2} \sin \left(\left(u_{2}-\beta\right) / 2\right) \cos u_{2}\right) \cdot(4.13 .1) \\
& +\left(u_{1}^{2} v_{1} v_{2} \sin u_{2}+u_{1}^{3} v_{2}^{2} \cos u_{2}-\mu \cos u_{2}-\frac{1}{2} \alpha u_{1}^{1 / 2} \cos \left(\left(u_{2}-\beta\right) / 2\right) \cos u_{2}\right. \\
& \left.+\alpha u_{1}^{1 / 2} \sin \left(\left(u_{2}-\beta\right) / 2\right) \sin u_{2}\right) \cdot(4.13 .2)+\left(-u_{1}^{2} v_{2} \cos u_{2}\right) \cdot(4.13 .3) \\
& +\left(-u_{1}^{2} v_{1} \cos u_{2}+2 u_{1}^{3} v_{2} \sin u_{2}\right) \cdot(4.13 .4)
\end{aligned}
$$

for $J_{2}$. Finally, $I$ comes from the combination

$$
\begin{aligned}
& \left(4 u_{1} v_{2} H+\frac{1}{2} u_{1}^{-1 / 2} v_{1} \alpha \sin \left(\left(u_{2}-\beta\right) / 2\right)\right. \\
& \left.+\frac{3}{2} u_{1}^{1 / 2} v_{2} \alpha \cos \left(\left(u_{2}-\beta\right) / 2\right)\right) \cdot(4.13 .1) \\
& +\left(\frac{1}{2} u_{1}^{1 / 2} v_{1} \alpha \cos \left(\left(u_{2}-\beta\right) / 2\right)-\frac{1}{2} u_{1}^{3 / 2} v_{2} \alpha \sin \left(\left(u_{2}-\beta\right) / 2\right)\right) .(4) \\
& +\left(u_{1}^{1 / 2} \alpha \sin \left(\left(u_{2}-\beta\right) / 2\right)\right) \cdot(4.13 .3) \\
& +\left(2 u_{1}^{2} H+u_{1}^{3 / 2} \alpha \cos \left(\left(u_{2}-\beta\right) / 2\right)\right) .(4.13 .4)
\end{aligned}
$$


wherein the fact that $d H=0$ is used. The lack of functional independence between $H, J_{1}, J_{2}$ and $I$ is implied immediately, since (4.13) can have only three independent characteristics.

\section{Discussion}

In the Appendix, a brief outline of the three-dimensional counterpart to the calculations of Section 2 is given. The result is that the only autonomous Hamiltonian of the form $H=T+V$ describing a three-dimensional motion which possesses a Laplace-Runge-Lenz vector (as distinct from a LaplaceRunge-Lenz-like vector) as defined in (1.6) is

$$
H=\frac{1}{2} \mathbf{p} \cdot \mathbf{p}-\mu / r,
$$

i.e. the standard Kepler problem. Thus it appears that the Hamiltonian (1.1) with its conserved vector (1.2) and cubic invariant (3.1) is an isolated occurrence. The conserved vector $\mathbf{J}$ is easily recognised as a generalisation of the standard Laplace-Runge-Lenz vector. As far as the cubic invariant $I$ is concerned, we regard it as a generalisation of the angular momentum. We note that, although the potential in (1.1) is not single-valued, Sen [11] went on to show that a consistent quantum-mechanical description of the problem for bound states can be made via the Casimir operator for $s o(3)$. In view of our equation (3.12), this should come as no surprise.

Finally, in Section 4 we demonstrated that all four integrals $\left(H, J_{1}, J_{2}\right.$ and $I$ ) can be obtained from the single symmetry of the equation of motion which was the generator of time translations, $\partial / \partial t$. We emphasise that, in an $N$-dimensional problem, with each generator there are associated $2 N-1$ independent first integrals, the characteristics of the equivalent of (4.5). We do not claim that it is a necessarily transparent process. Indeed, it may not be possible to obtain them in global closed form, but then that is always a hazard associated with the solution of systems of first-order equations.

\section{Acknowledgements}

Both of us acknowledge the support of the Foundation for Research Development of South Africa, and one of us (VMG) the support of the University of the Witwatersrand, Johannesburg, through a Postgraduate Bursary.

\section{Appendix}

For the three-dimensional problem, the equations corresponding to (2.1-3) are, in spherical polar co-ordinates,

$$
H=\frac{1}{2}\left(p_{r}^{2}+\frac{p_{\theta}^{2}}{r^{2}}+\frac{p_{\phi}^{2}}{r^{2} \sin ^{2} \theta}\right)+V(r, \theta, \phi),
$$




$$
\begin{aligned}
J_{1}= & \frac{1}{r}\left(p_{\theta}^{2}+\frac{p_{\phi}^{2}}{\sin ^{2} \theta}\right) \sin \theta \cos \phi-p_{r} p_{\theta} \cos \theta \cos \phi \\
& +\frac{p_{r} p_{\phi}}{\sin \theta} \sin \phi+f(r, \theta, \phi), \\
J_{2}= & \frac{1}{r}\left(p_{\theta}^{2}+\frac{p_{\phi}^{2}}{\sin ^{2} \theta}\right) \sin \theta \sin \phi-p_{r} p_{\theta} \cos \theta \sin \phi \\
& -\frac{p_{r} p_{\phi}}{\sin \theta} \cos \phi+g(r, \theta, \phi), \\
J_{3}=\frac{1}{r} & \left(p_{\theta}^{2}+\frac{p_{\phi}^{2}}{\sin ^{2} \theta}\right) \cos \theta+p_{r} p_{\theta} \sin \theta+h(r, \theta, \phi) .
\end{aligned}
$$

The requirement that $J_{1}, J_{2}$ and $J_{3}$ have zero Poisson bracket with $H$ leads to nine equations for the $r, \theta$ and $\phi$ derivatives of $f, g$ and $h$. The imposition of the consistency condition between the mixed derivatives of $f, g$ and $h$ leads to nine equations for the potential $V$. These can be rearranged to give the simpler equations

$$
\begin{gathered}
\frac{\partial}{\partial r}\left(r^{2} \frac{\partial V}{\partial \phi}\right)=0, \\
\frac{\partial}{\partial r}\left(r^{2} \frac{\partial V}{\partial \theta}\right)=0, \\
\frac{\partial}{\partial \theta}\left(\frac{1}{\sin \theta} \frac{\partial V}{\partial \phi}\right)=0, \\
\frac{\partial}{\partial r}\left(r^{2} \frac{\partial V}{\partial r}\right)-\frac{\partial^{2} V}{\partial \theta^{2}}=0, \\
\frac{\partial}{\partial r}\left(2 r \frac{\partial V}{\partial \theta}\right)-\frac{\partial V}{\partial \theta}=0, \\
\frac{\partial}{\partial r}\left(2 r \frac{\partial V}{\partial \phi}\right)-\frac{\partial V}{\partial \phi}=0, \\
\sin \theta\left[\frac{\partial}{\partial r}\left(r^{2} \frac{\partial V}{\partial r}\right)-\frac{1}{\sin ^{2} \theta} \frac{\partial^{2} V}{\partial \phi^{2}}\right]-\cos \theta \frac{\partial V}{\partial \theta}=0
\end{gathered}
$$

(two equations become identically zero). The only nontrivial solution to (A5) is

$$
V=-\frac{\mu}{r},
$$

which is the potential for the standard Kepler problem. 


\section{References}

[1] C. D. Collinson, "Investigation of planetary orbits using the Lenz-Runge vector", Bull. Inst. Math. Applics. 13 (1973) 377-378.

[2] D. M. Fradkin, "Existence of the dynamic symmetries $\mathrm{O}_{4}$ and $\mathrm{SU}_{3}$ for all classical central potential problems", Prog. Theor. Phys. 37 (1967) 793-812.

[3] Herbert Goldstein, "Prehistory of the 'Runge-Lenz' vector", Am. J. Phys. 43 (1975) 737738.

[4] Herbert Goldstein, "More on the prehistory of the Laplace or Runge-Lenz vector", Am. J. Phys. 44 (1976) 1123-1124.

[5] V. M. Gorringe and P. G. L. Leach, "Conserved vectors for the autonomous system $\ddot{\mathbf{r}}+$ $g(r, \theta) \hat{\mathbf{r}}+h(r, \theta) \hat{\theta}=0$ ", Physica 27D (1987) 243-248.

[6] L. S. Hall, "A theory of exact and approximate configurational invariants", Physica 8D (1983) 90-113.

[7] P. G. L. Leach, “A further note on the Hénon-Heiles problem”, J. Math. Phys. 22 (1981) 679-682.

[8] P. G. L. Leach, "Applications of the Lie theory of extended groups in Hamiltonian mechanics: the oscillator and the Kepler problem", J. Austral. Math. Soc. (Ser B) 23 (1981) 173-186.

[9] P. G. L. Leach and V. M. Gorringe, "Variations on Newton's Keplerian theme", S. A. J. Sci. 83 (1987) 550-555.

[10] P. G. L. Leach and V. M. Gorringe, "A conserved Laplace-Runge-Lenz-like vector for a class of three-dimensional motions", Phys. Lett. 133A (1988) 289-294.

[11] G. E. Prince and C. J. Eliezer "On the Lie symmetries of the classical Kepler problem", $J$. Phys. A 14 (1981) 587-596.

[12] W. Sarlet, P. G. L. Leach and F. Cantrijn, "First integrals versus configurational invariants and a weak form of complete integrability", Physica 17D (1985) 87-98.

[13] Tanaji Sen, “A class of integrable potentials", J. Math. Phys. 28 (1987) 2841-2850.

[14] G. Thompson, "Second-order systems with Runge-Lenz-type vectors", Lett. Math. Phys. 14 (1987) 69-75.

[15] E. T. Whittaker, "A treatise on the analytical dynamics of particles and rigid bodies", (Dover, New York, 1944) p. 331.

[16] Whittaker op cit page 320 gives the reference S. D. Poisson, $J$ de l' École Polyt. 8 (1809) 266.

[17] Takeshi Yoshida "Determination of the generalized Laplace-Runge-Lenz vector by an inverse matrix method", Am. J. Phys. 57 (1989) 376-377. 